\title{
COMPUTER SPATIALLY ORIENTED RECONSTRUCTION OF A 3D HEART SHAPE BASED ON ITS TOMOGRAPHIC IMAGING
}

\author{
Natalya Natalinova ${ }^{a}$, Diana Avdeeva, Veniamin Kazakov, Vladimir Baranov, Olga Galtseva, \\ Denis Ivashkov
}

Tomsk Polytechnic University, 634050, Tomsk, Russia

\begin{abstract}
Diagnostics of cardiovascular system conditions and diseases is considered the most important task of electrocardiology. The aim of the study is to define geometric parameters of the patient heart and the synthesis of a realistic three-dimensional heart image based on a series of two-dimensional images obtained by computer tomography. The preparation and further processing of medical image data is an important initial step for further study of the heart electrodynamic activity. The problem is solved with the help of computer tomography method by preparing a series of images of the patient heart. The technique of volumetric rendering is applied to represent certain anatomical structures in a three-dimensional (3D) graphical form. It is concluded that the character of the obtained three-dimensional model of the patient heart is determined by the quality of the input data, the resolution of the tomograph, the tomographic slice thickness, the accuracy of the object boundaries determining the segmentation process and the peculiarities of medical image processing by the software applied.
\end{abstract}

\section{Introduction}

The cardiovascular system is regarded as a complex multi-level mechanism in its functioning. Thus, a problem of ensuring an accurate diagnosis might often be solved only by experienced medical specialists. This study was conducted as part of the development of non-invasive methods of diagnosis causes of sudden cardiac death. One reason is presumed to be the presence of a potential delayed depolarization of the heart ventricles. The research objective is to develop a computer model of the heart, which in combination with the highly sensitive ECG measurement methods can be used to diagnose potential delayed depolarization of the ventricles of the heart. A creation of numerical model of a heart will allow solving the inverse problem of reconstructing the characteristics of cardiac tissue on the basis of the ECG signal with the high-resolution data in the hearts and computed tomography of the heart geometry. The virtual model heart application is a visual representation of the cardiovascular system and enables the doctor-diagnostician to compare the electrocardiogram (ECG) changes with the damaged myocardial tissue on the realistic three-dimensional heart images, in particular, at the level of microvolts. Therefore

\footnotetext{
${ }^{a}$ Corresponding author : natalinova@tpu.ru
} 
there is a vital need to obtain an apparent three-dimensional visualization of the patient heart for further research of the electrodynamic heart activity by means of the finite element method of CAD software [1,2].

The paper aims to define geometric parameters of the patient heart and the synthesis of a realistic three-dimensional heart image based on a series of two-dimensional images obtained by computed tomography. Thus, obtaining and further processing of medical image data is viewed as an important step for further research of electrodynamic heart activity.

Figure 1 shows the algorithm for obtaining a 3D heart model.

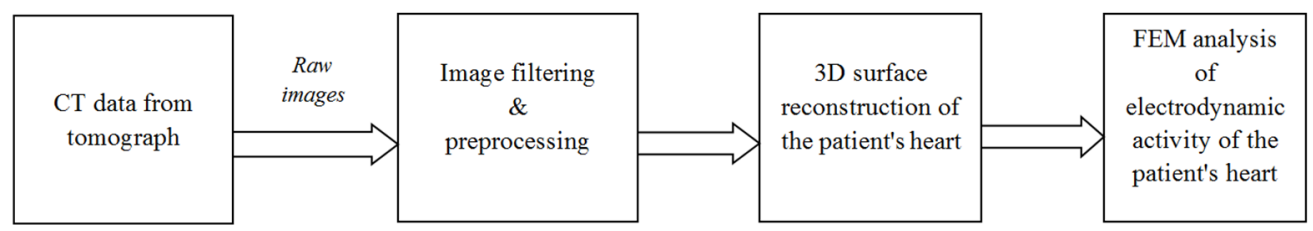

Figure 1. Processing of medical imaging data based on computed tomography slices.

\section{Method of 3D reconstruction of tomographic images}

The initial information obtained by computer tomography is a sequence of two-dimensional (2D) digital images. Each pixel in a 2D computed tomography (CT) image is a function of X-ray density of the object of study at the corresponding point $\mathrm{q}(\mathrm{x}, \mathrm{y}, \mathrm{z})$, represented in gray scale. These images are stored and transmitted as Digital Imaging and Communications in Medicine (DICOM) file format series. Tomographic slices have an equal thickness and the same number of pixels per slice. The tomographic slices are further ordered; there is created the volume image represented by a regular grid of voxels in the diagnostic software (tomographic reconstruction) which is characterized by different absorption intensity and reflects the structure of the research object. Figure 2 shows the series of 2D images of the patient's heart obtained from CT. The visual representation of individual anatomical structures in virtual three-dimensional objects is obtained by a $3 \mathrm{D}$ volume rendering technique. For this purpose the operator determines the threshold X-ray density (e.g., those corresponding to the density of the bone tissue, or cardiac tissue).
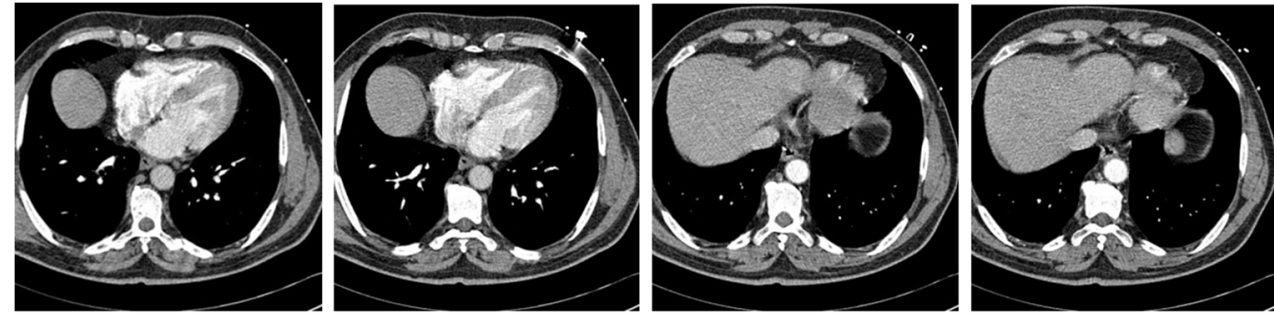

Figure 2. Series of 2D images of the patient's heart obtained from CT.

The three-dimensional model is then built according to a given range of X-ray density in the software. There exist numerous image processing packages, either commercial (Amira, Visage Imaging; MeVisLab, Mevis; Imaris, BitPlane) or open source (VOXX, 3D Slicer, BioImage XD, etc.). These packages offer excellent solutions for the specific problems they were designed to solve [1].

To understand the process of three-dimensional image reconstruction, consider the algorithm which is used in the software 3D Slicer. By changing the threshold density X-ray techniques and graphic image processing, it is possible to create a complex, multi- 
component model consisting of individual elements, which correspond to the bones, muscles, lungs, blood vessels, etc. The method of Marching cubes is used to transform a set of voxels into the polygonal model as the standard mathematical algorithm. Some 3D models are emphasized by different colors and transparency changes for highest visibility and easy image perception of the research object.

\section{Results}

Figure 3 shows the logical structure of the software algorithm implementing the numerical model of the heart, which allows to solve the inverse problem of reconstructing the characteristics of cardiac tissue on the basis of the ECG signal data and high-resolution computed tomography of the heart geometry. Software consists of the following modules: 1 - the module of geometric data preparation, 2 - the module of heart bidomain model configuration, 3 - the model of numerical modelling on base of the obtained heart bidomain model configuration, 4 - the control module which determines to continue to seek the error minimum, or to stop, 5 - the module of error calculation between heart model ECG and realistic ECG with the high-resolution [3-5].

Input

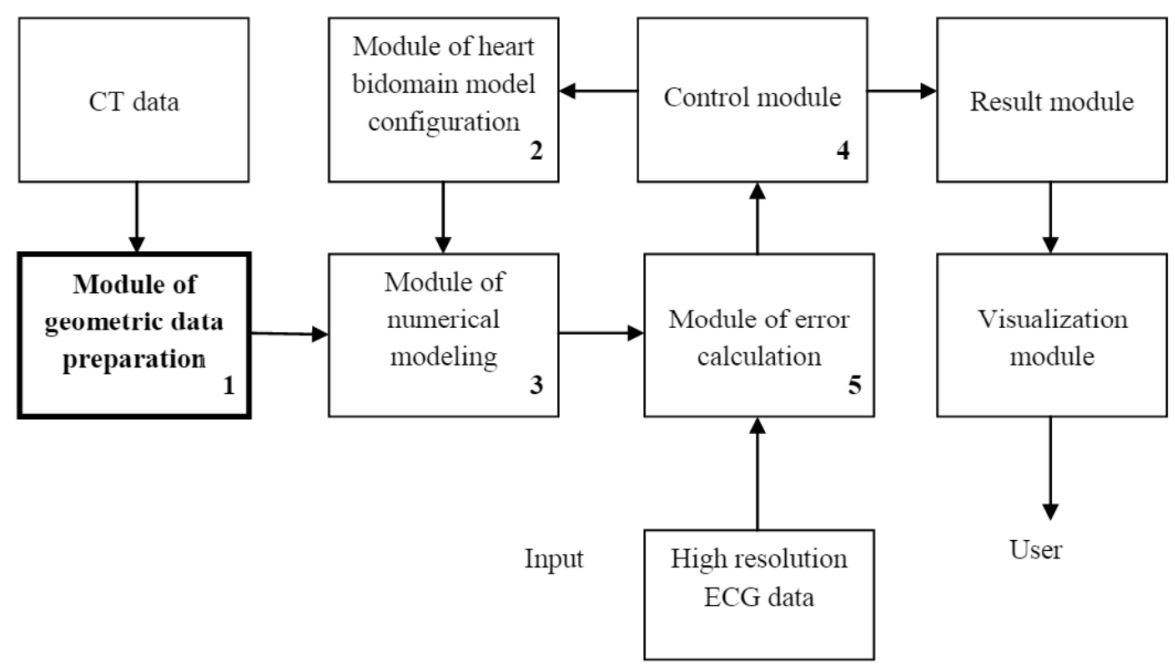

Figure 3. The logical structure of the algorithm.

The input data for the program are the data of the patient CT tomograph and an ECG signal obtained via a hardware software system for measuring heart micropotential. The creation of three-dimensional model of the patient's heart (preparation of the geometry data) is a preparatory stage for the diagnosis of heart disease at an early stage .

The first step of the process of creating a three-dimensional model of the patient heart covered the uploading of the tomographic images in DICOM format. Then the range of gray shades corresponding to the density of the heart was selected, and, finally, a geometric model was reproduced based on these data, Figure 4 shows the resulting image of the individual realistic model of the patient heart. 

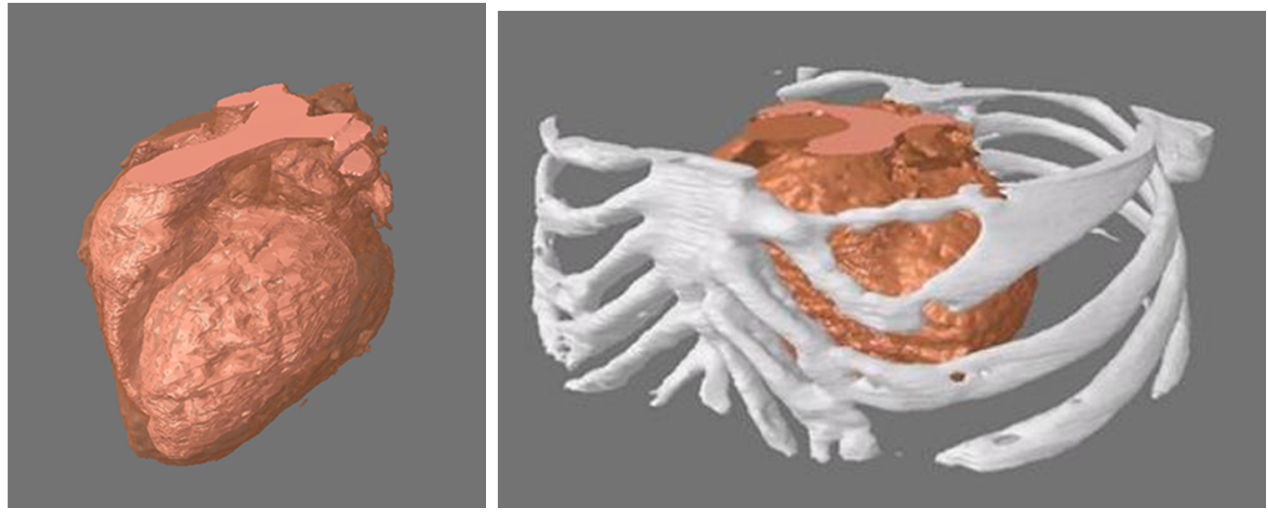

Figure 4. An individual realistic model of the patient heart.

The resulting 3D virtual model of all the elements of the patient torso areas were removed not to effect further calculations of electrodynamic heart activity. The resulting model was saved in the STL format, which allowed importing it into the CAD complex "Chaste» $[2,11]$ for further analysis by the finite element method.

The quality of the resulting model is determined by the quality of the input data resolution images, the tomographic slice thickness, the precision of the object boundary definition during the segmentation process, some peculiarities of the model handling features in the medical imaging software [6-12].

The great importance in the process of creating virtual 3D-models is a preliminary assessment of the tomographic image quality, the identification of existing artifacts and their further elimination in the process of 3D rendering. The quality of the resulting model is determined by several features, such as: the quality of input data resolution of a tomograph, the tomographic slice thickness within the definition of the research object boundaries in the segmentation process, model handling features in software for medical imaging. Although a computer tomography is a precise method of medical research, its implementation might be affected by various artifacts, which in their turn can significantly influence the quality of the resulting 3D-model. Among the artifacts there are the following ones:

- linear artifacts that occur near objects which absorb most of the x-rays;

- the effect of the image blurring in the sharp edge areas and abrupt transition zone from high to low X-ray density. To reduce this negative effect it is possible to increase a number of sensors and decrease the tomographic slice thickness.

- occurrence of one or more "rings" in the image. This effect is usually associated with mechanical breakdown of the detector;

- "a noise artifact" that shows grainy images. This effect is usually associated with the low ratio of signal and noise or insufficient capacity of an X-ray tube;

- artifacts associated with the movement of the research object, appear blurred or with linear distortions. These artifacts are minimized in modern tomographs by means of reducing the time spent on processing a single slice.

- "steps" in the images. This effect is usually associated with large thickness of the tomographic slice, etc. [11].

Obtained geometrical model is a part of the software designed for the diagnosis of lowamplitude biopotentials of the heart, such as ventricular late potentials (VLP), arising due to delayed depolarization of the ventricles of the heart. The heart electrodynamic model allows solving the problem of detection and localization of necrotic tissue sections of heart, leading to the appearance of low-amplitude high frequency components in the ECG signal 
by varying the size distribution and the characteristics of the conductivity tensor of the transmembrane action potential.

This computer heart model is combined with highly sensitive methods of ECG measurement and it is part of the hardware-software complex diagnostic potential delayed depolarization of the ventricles of the heart.

\section{Conclusion}

Image processing system with modeling of 3D patient's heart makes possible to get comprehensive information on the condition of the cardiovascular system, its shape, a location and geometric dimensions. A computer model of a 3D heart shape based on its tomographic imaging duplicates the human heart as accurate as possible. The computer model of the heart provides an opportunity for further study of the electrodynamic activity of the heart using finite element method based on the anatomical characteristics of a particular patient's heart. Thus, creating a heart model is an important step in the process of non-invasive method of heart disease diagnostics. Due to this, scientists will be able to determine abnormalities in the heart, even at early stages and early diagnosis of ordinary citizens to reduce the number of severe and fatal outcomes of chronic heart disease.

\section{Acknowledgment}

The research was financially supported by the Federal Targeted Programme "Research and Development in Priority Fields of S\&T Complex of Russia in 2014-2020", the Agreement No. 14.578.21.0032 dated 05.06.2014 "Development of the experimental sample of a hardware-software complex for noninvasive recording of heart micropotentials in a wide frequency band without filtering and averaging in real time to early detect the symptoms of sudden cardiac death", the unique identifier of the contract: RFMEF157814X0032.

\section{References}

[1] S. Sovilj, R. Magjarevic, N. Lovell, S. Dokos, CinC 40, 377 (2013)

[2] J. Pitt-Francis, P. Pathmanathan, M.O. Bernabeu, R. Bordas, J. Cooper, A.G. Fletcher, G.R. Mirams, P. Murray, J.M. Osborne, A. Walter, S.J. Chapman, A. Garny, I.M.M. van Leeuwen, P.K. Maini, B. Rodriguez, S.L. Waters, J.P. Whiteley, H.M. Byrne, D.J. Gavaghan, Comput. Phys. Commun. 180, 2452 (2009) doi: 10.1016/j.cpc.2009.07.019

[3] V. Y. Kazakov, D. K. Avdeeva, M. G. Grigoriev, N. M. Natalinova, I. V. Maksimov, M. V. Balahonova, BLM 7, 1 (2015)

[4] D. K. Avdeeva, V. Y. Kazakov, N. M. Natalinova, M. L. Ivanov, M. A. Yuzhakova, N. V. Turushev, Eur. J. Phys. and Health Educ. 6, BM-015-14 (2014)

[5] D. K. Avdeeva, V. Y. Kazakov, N. M. Natalinova, I. V. Maksimov, M. V. Balahonova, BLM 6, BM-025 (2014)

[6] P. Pathmanathan, R.A. Gray, Int J Numer Method Biomed Eng. 30, 525 (2014) doi: $10.1002 / \mathrm{cnm} .2615$

[7] S.A. Niederer, A.K. Shetty, G. Plank, J. Bostock, R. Razavi, N.P. Smith, C.A. Rinaldi, PACE 35, 204 (2012) doi: 10.1111/j.1540-8159.2011.03243.x

[8] D. Noble, A. Garny, P.J. Noble, J Physiol. 590, 2613 (2012) doi: 10.1113/jphysiol.2011.224238

[9] V. N. Borikov, P. F. Baranov, A. D. Bezshlyakh, IEEE SIBCON-2009, 5044870 (2009) 
[10] X.H. Wang, X.Y. Li, X.J. Zhang, IFMBE Proc. 31 IFMBE, 1503 (2010) doi: 10.1007/978-3-642-14515-5 383

[11] G.R. Mirams, C.J. Arthurs, M.O. Bernabeu, R. Bordas, J. Cooper, A. Corrias, Y. Davit, S.-J. Dunn, A.G. Fletcher, D.G. Harvey, M.E. Marsh, J.M. Osborne, P. Pathmanathan, J. Pitt-Francis, J. Southern, N. Zemzemi, D.J. Gavaghan, PLoS Comput. Biol. 9 (3), e1002970 (2013) DOI: 10.1371/journal.pcbi.1002970

[12] E.J. Vigmond, M. Hughes, G. Plank, L.J. Leon, J Electrocardiol. 36, 69 (2003) doi: 10.1016/j.jelectrocard.2003.09.017 